\title{
Experimental Issues in High-Sensitivity Charm Experiments
}

\author{
J.A. Appel \\ Fermi National Accelerator Laboratory \\ P.O. Box 500, Batavia, Illinois 60510
}

July 1994

Presented at CHARM2000 Workshop, June 7, 1994. 


\section{Disclaimer}

This report was prepared as an account of work sponsored by an agency of the United States Government. Neither the United States Government nor any agency thereof, nor any of their employees, makes any warranty, express or implied, or assumes any legal liability or responsibility for the accuracy, completeness, or usefulness of any information, apparatus, product, or process disclosed, or represents that its use would not infringe privately owned rights. Reference herein to any specific commercial product, process, or service by trade name, trademark, manufacturer, or otherwise, does not necessarily constitute or imply its endorsement, recommendation, or favoring by the United States Government or any agency thereof. The views and opinions of authors expressed herein do not necessarily state or reflect those of the United States Government or any agency thereof. 


\author{
Jeffrey A. Appel \\ Fermi National Accelerator Laboratory* \\ CHARM2000 Workshop
}

June 7, 1994

\begin{abstract}
Progress in the exploration of charm physics at fixed target experiments has been prodigious over the last 15 years. The issue before the CHARM2000 Workshop is whether and how this progress can be continued beyond the next fixed target run. An equivalent of $10^{8}$ fully reconstructed charm decays has been selected as a worthy goal. Underlying all this is the list of physics questions which can be answered by pursuing charm in this way. This paper reviews the experimental issues associated with making this next step. It draws heavily on the experience gathered over the period of rapid progress and, at the end, poses the questions of what is needed and what choices may need to be made.
\end{abstract}

*Work supported by the U.S. Department of Energy under contract No. DE-ACO2-76CHO3000. 


\section{Progress and Projections}

One measure of the progress in charm physics over the last 15 years is the number of charm decays fully reconstructed by a single experiment. In 1980, a fixed target experiment was lucky to observe one hundred such decays. Many experiments before had actually failed to produce significant signals at all. However the real progress has come since that time. Figure 1 demonstrates the exponential growth in Fermilab fixed target charm samples since 1980.[1] The rate of growth has averaged 1.7 per year, a factor of 5 per running period. This growth is projected to continue at about the same rate into the next running period also.

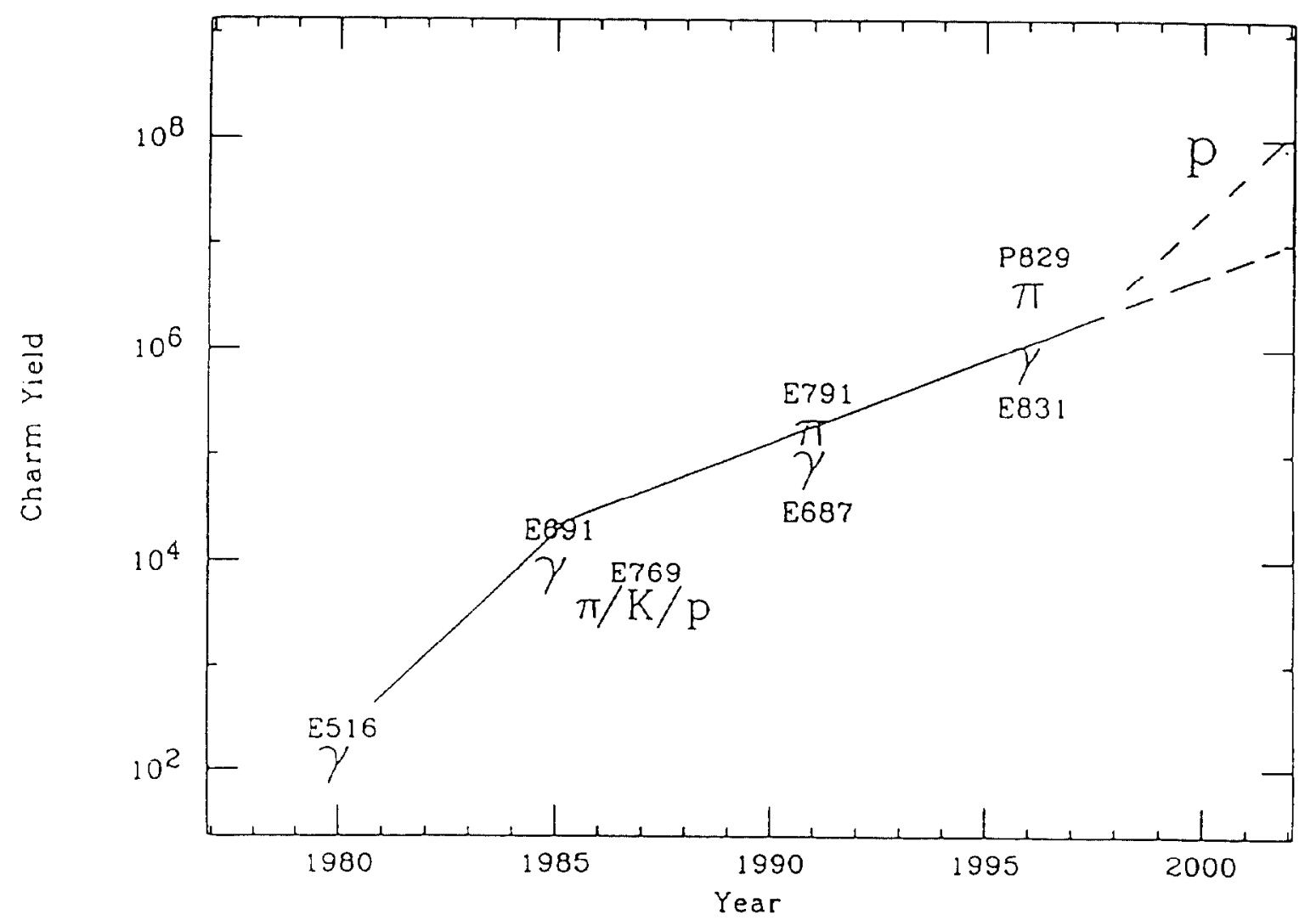

Fig. 1. Progression of the number of reconstructed charm decays in Fermilab fixed-target experiments.

The announced goal of the CHARM2000 Workshop is $10^{8}$ fully reconstructed charm decays. As can be seen in Fig. 1, this requires a step as great as the most aggressive single step achieved in the history of the last 15 years.

\section{New Techniques Along the Way So Far - Critical Issue}

The single biggest step in the progress shown in Fig. 1 appeared with the ability to do precision vertex determination combined with the ability to handle vastly increased amounts of data[2] These advances were made possible by the introduction of silicon microstrip detectors (SMDs) and parallel processing farms of inexpensive computers. These, in turn, allowed the 
experimenters to take advantage of the good duty factor and high intensity, high energy beams of the Tevatron accelerator. The rather open on-line event selection is what resulted in an impressive range of physics capability, and is why the CHARM2000 goal is stated in terms of fully reconstructed charm decays.

The size of the biggest step in increased charm capability came mostly because of the silicon microstrip detectors. These have been widely recognized as the important technological innovation making high statistics charm experiments possible in a fixed target environment. The precision tracking of particles with the SMDs is used in the separate identification of the primary interaction vertex and the charm particle decay vertex. This, in turn, has two features which are both essential in improving capability: first, the selection of events which have a high probability of charm in them and second, the identification of that subset of tracks coming from a charm decay. Both features assist in improving the signal over background. The latter feature is specially critical in hadroproduction where the total number of tracks in a charm event is quite high.

In spite of the importance of the SMDs, additional technological innovation has been needed. This additional innovation was primarily associated with handling ever increasing amounts of data. Off-line computer power increased about as fast as the increase in charm samples. However, it has also been necessary to speed up the front end signal processing, to increase data readout rates, to add on-line data storage, as well as to increase long term data storage density and cost effectiveness. These features have been the primary engines of increased physics capability since the introduction of the SMDs.

The list of technological advances has been quite long. All of the advances have been necessary in order to maintain the exponential growth of the number of reconstructed charm decays. The reach of the next fixed target run depends on applying these same set of improvements. However, aside from the anticipated reduction in the per-calculation-cost of offline computing, no new technology has yet been identified for the next run. What can one say about the period beyond the next fixed target run? Some new technology will need to be applied to continue the current rate of improvement. Some extra ordinary improvement will be needed to reach $10^{8}$ reconstructed charm decays.

\section{Other Relevant Experimental Issues}

Beyond the critical issue of technological innovation, there exists a rather long list of choices to be made in preparing an experiment. These choices may be influenced, even driven, by any technological innovation planned. Failing to have identified an obvious such innovation, it is still possible to review the issues which have been important so far. These had best be considered when an experiment is conceptualized.

It is important to remember that an experiment is always a compromise among divergent pulls. Yet, one often hears that a particular factor is enough to justify proposing a new effort. In fact, it is the combination of choices which must work together to achieve an experimental goal. Thus, no one of the following set of issues can be viewed independently of the others. They each play a role in determining how well an experiment can do in rcaching toward a particular goal. And, the particularity of the goal matters. Different goals (at a minimum, different final decay modes of a particular charm particle) will have different benefits from any experiment configuration choice. There is no choice which is best for everything. 


\subsection{Cross Sections and Rate Projections}

By now, cross sections for the common charm particles as seen in particular decay modes are well measured by multiple experiments. In photoproduction, the cross section is forward peaked (typically reaching a maximum at Feynman $x$ of 0.2 ) and the cross section is slowly saturating at the energies achievable with today's Tevatron.[3] In hadroproduction, the cross section is still rising at Tevatron energies, growing by a factor of two for each factor of two in available beam energy. [4] The mesons are produced more centrally and, for protons, are symmetric about Feynman $x$ of 0.0. The falloff with Feynman $x$ is quite steep, although leading particle effects may be useful for restricted classes of charm studies (e.g., baryon physics as proposed for E781). The total cross section for photoproduction reaches about 1 microbarn, while for forward hadroproduction (Feynman $x>0.0$ ) the cross section is more like 20 microbarns.

\subsection{Incident Particles}

While the cross section for charm production is essential for reaching the $10^{8}$ level of reconstructed charm decays, the cross section is not the only feature of importance related to the incident particle type. The track multiplicity in events, for example, and the resulting signal to background are important. After all, the reason to seek $10^{8}$ decays is to reach more rare occurences, and backgrounds must be reduced to make the signals useful for physics.

Photoproduction and hadroproduction track multiplicities are shown in Fig. 2. The most likely number of tracks in photoproduction E691 was 10, while in hadroproduction E791 the value is about 14. Even among the hadroproduction beam options, there are differences. Signal to background is somewhat worse for protons than for pions and kaons, even after selecting optimal cuts. Of course, the event selection for proton induced events is more demanding and the efficiency is lower - just as was the case in going from photoproduction to hadroproduction and achieving the same signal to background levels. There is about a factor two lower acceptance in hadroproduction relative to photoproduction when the same signal to background is required.
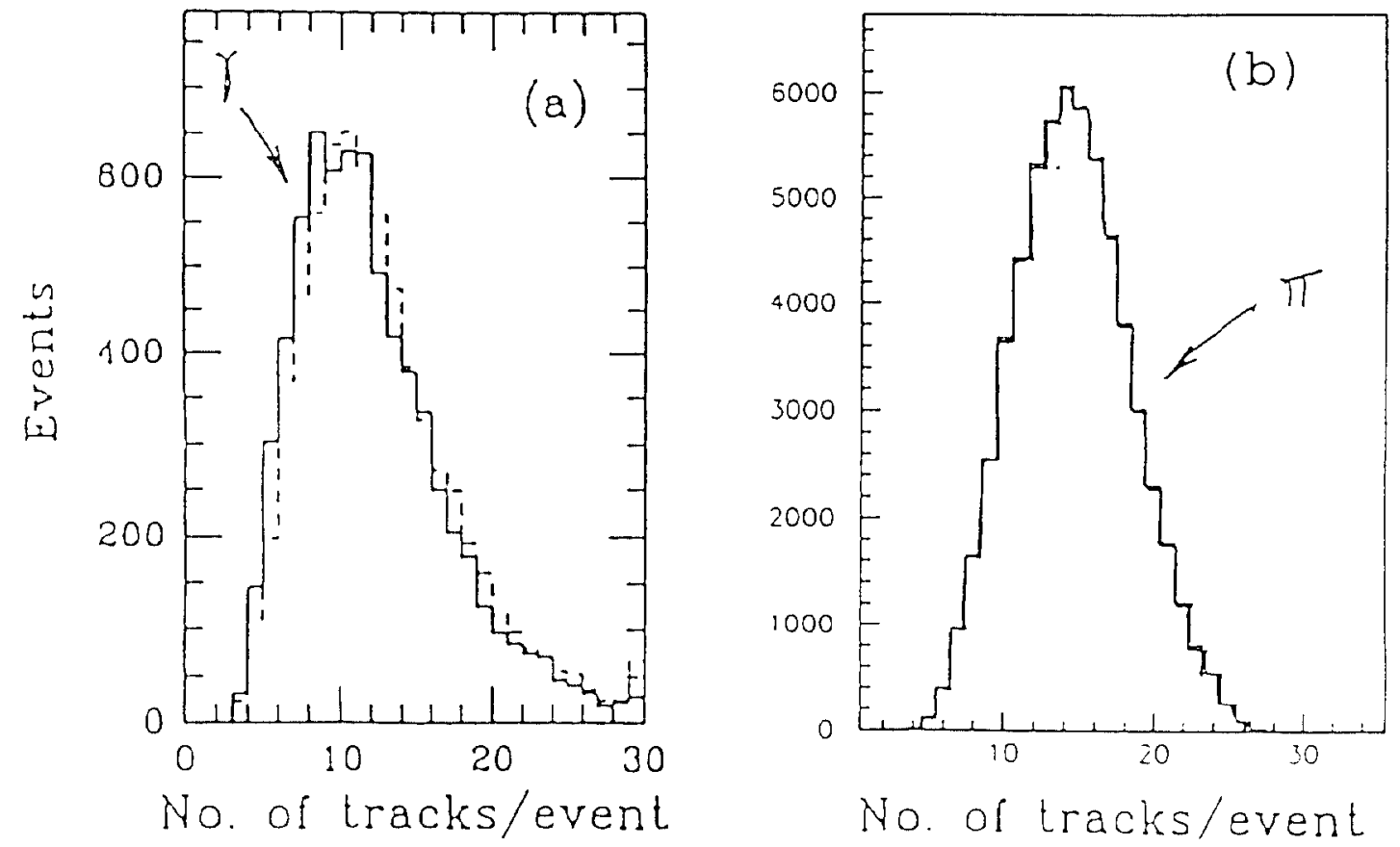

Fig. 2. Charged track multiplicity in (a) photoproduced charm events and (b) hadroproduced charm candidate events. 


\subsection{Event Topologies}

The cloud of particles produced in the violent interactions which produce charm may be a problem in terms of event reconstruction complexity and backgrounds. However, there is a silver lining to that cloud. In particular, the higher average track multiplicity is useful in identifying the primary interaction point. The precision with which that vertex can be specified is also improved. Having an incident high momentum track is also useful in specifying the transverse location of the primary interaction.

\subsection{A Dependence and Other Target Material Issues}

Heavy nuclear targets appear beneficial in rate calculations since the charm production rate is roughly proportional to the atomic weight of the target, $A$. On the other hand, heavy targets are ruled out for photoproduction (due to pair conversions of the beam). In hadroproduction, there are a number of penalties paid for a heavy target. These include (1) higher track multiplicity with the concomitant worse signal to background for a given set of cuts, (2) lower efficiencies for most signals at the optimal cuts, (3) possibly worse mass resolution (certainly when decays are allowed in the target or when multiple target foils are used).

\subsection{Decays in Free Space}

The importance of secondary interactions as a source of backgrounds for charm signals has become increasingly apparent to experimenters. Candidate charm decays appear at downstream locations where there is material. E687 is showing generic charm generated Monte Carlo events which model the wings of mass distributions only when the decays are selected as appearing in free space. [5] Otherwise (i.e., when vertices are allowed to appear in matter), the backgrounds are significantly worse.

\subsection{Acceptance Issues}

In designing an experiment, a kinematic region for accepted events is selected consciously or unconsciously. This selection has implications for (1) the rates of reconstructed decays, (2) the resolutions of mass and other parameters and (3) the signal to background ratios. Typically, where the rates are smaller, the signals are cleaner. Thus, less background is generally evident in plots at higher Feynman $x$ and at higher transverse momentum. On the other hand, since higher Feynman $x$ corresponds to higher decay particle momenta, the mass resolution is surprisingly worse at high Feynman $x$. In E791, for example, the mass resolution at Feynman $x$ of 0.8 is three times worse than at the optimal 0.1. Multiple scattering dominates only below this value of Feynman $x$. This may come as a surprise to people used to $e^{+} e^{-}$environments.

There is a little noticed implication of acceptance for mixing searches in $D^{0}$ hadronic decays. The acceptance as a function of proper lifetime is a function of the selection criteria used. The more selective one becomes, as required to push mixing limits further, typically the more reduced is the efficiency for short lifetimes. On the one hand, this helps in getting away from the background posed by doubly Cabibbo suppressed decays which have an exponential dependence on proper lifetime. The mixing signal is expected to be maximal at two proper lifetimes. On the other hand, once one allows for interference between the doubly Cabibbo suppressed decay amplitude and the mixing amplitude, one needs acceptance in the short lifetime regime as well, in 
order to separate the two possible sources of decay. Doubly Cabibbo suppresed decays are background to the much more interesting possibility of $D^{0}$ mixing.

In the same way that inefficiency at shorter lifetimes affects the $D^{0}$ mixing capability of an experiment, it also influences the capability of observing one charm particle relative to another. Since tighter cuts are required in charm hadroproduction than in photoproduction, the capability of hadroproduction for charm mesons will be optimal relative to photoproduction. For the shorterlived charm baryons, on the other hand, hadroproduction experiments will have reduced efficiency.

\section{Trigger, Data Acquisition, and Off-Line Computing Issues}

While trigger, data acquisiton and off-line computing has been made a separate section of this review, it is really a continuation of the previous section on experimental issues. Choices of experimental configuration have a direct effect on triggering, data acquisition and off-line computing. It would be better to think of the whole configuration together. Restricting acceptance of the detector to particular regions of phase space or particle types directly affects triggering and is a part of it. The choice of very wide acceptance and the selection of more complicated events directly influence the computing load of the experiment, both on-line and off-line.

\subsection{Triggers - Event Selection Issues}

The main goal of the experiment trigger is to enrich the charm fraction of the sample of events which enter the data acquisition stream and pass along it. This enrichment, $E$, is the product of the rejection of unwanted events, $R$, and the efficiency of the trigger for the events of interest, eff.

$$
E=R * e f f
$$

The goal is to optimize $E$, not $R$ or eff alone. The associated issues include the level of sophistication required and the level of complexity needed. Somewhere among these words should be read cost and difficulty.

Recent fixed target triggers for charm have been notable for their directness, simplicity, and openness. This is in distinction to the first charm experiment at the Tagged Photon Laboratory, E516. In that case, a very fast and sophisticated system (the ECL-CAMAC Trigger Processor [6]) was designed and built to select events with large forward going effective mass, based on measurements of recoiling protons alone. The system worked very well technically. However, it failed to enrich the sample of recorded events. The measurement was indirect and the efficiency for the average charm particle was low. The more open triggers in use now either accept almost all hadronic interaction events or select those events with high forward effective mass (approximated by transverse energy) measured directly by observing the relevant particles themselves.

The options for selecting charm events at the trigger level comprise a reasonably long list. A version of the list presented in 1981 at Erice is shown in Table I. As far as I am aware, this was the first time that transverse energy was suggested as a trigger for charm in fixed target experiments. [7] Missing from the Erice trigger list is direct observation of evidence for secondary charm decay vertices. Many have proposed, and recently experiments at CERN and E789 at Fermilab have used sophisticated triggers looking for such evidence on-line. These triggers are the most direct charm cuts one can use on-line. They are similar to the important off-line analysis selection cuts for charm events used by all. However, two things should be noted. Even having 
the full reconstruction of events with final calibrations of the detector, early-selection subsets of data have only been reduced by typical factors of 5-10 using this kind of information. The smallness of this factor may be due, in part, to the ability to handle large data sets and to a natural conservatism.

Table I.

\section{Trigger Possiblities}

A. Target Recoil

1. High Forward Mass

*a. Missing Mass à la TPL

b. $T>T_{M I N}$ or Recoil $K E>K E_{M I N}$

2. Coherence of Scattering From Nucleus

a. For later reconstruction

b. Primakoff trigger for $\eta$

B. Decay Product

$$
c, b \ldots
$$

1. $\operatorname{High} p_{T}$

*a. Leptons

b. Charged particles

2. Decay Chain

a. $K^{ \pm}$in Cerenkov/momentum correlation

b. $K^{\mathrm{o}}, \Lambda^{\mathrm{o}}$ - Downstream $V^{\mathrm{o}}$ or $\Delta Q$

c. $\pi \pm$ from $D^{*} \rightarrow D \pi$

d. $\Delta Q^{\prime}$ s near target

$$
B * * \rightarrow B \pi
$$

e. $\quad v$ - missing energy

C. Event Topology

1. Multipicity

2. $\Sigma\left|p_{T}\right|, \Sigma p_{T}^{2}, \Sigma w^{2}$ from calorimeters 
However, it will take courage to reject larger factors on-line than experiments have been willing to reject off-line. The largest rejection which can be obtained reasonably with a transverse energy requirement on-line is about 5 . In conjunction with such a transverse energy cut, the extra rejection of a secondary vertex trigger will be worse. The larger factors of rejection sometimes cited also fail to maintain efficiency for final charm samples. The factors of enrichment which one reads in the literature are usually for the longest-lived charm particles only.

\subsection{Data Acquisition Issues}

Most recently at Fermilab, experiments have taken to increasingly powerful data acquisition systems and recording increasingly large data sets. This has been made possible by improvements in electronic circuits, to be sure, but mostly by the growth of parallelism and the cost effectiveness of writing data to $8 \mathrm{~mm}$ magnetic tape. Table II demonstrates this pattern from the experiments at the Tagged Photon Laboratory. The data set sizes have grown by a factor of 700 in the decade of the 80 's, the number of events by 1000 . The number of reconstruced charm decays in final data sets has grown by even more, a factor of about 2000 . A continuation of this trend requires technology beyond what is presently forseeable. Thus, there is great interest in improving triggers and/or moving more computing on-line in the future.

Table II.

\begin{tabular}{|cccccccc|}
\hline \multicolumn{7}{c|}{ Growth of DA Parallelism } \\
\hline \hline $\begin{array}{c}\text { Time } \\
\text { Frame }\end{array}$ & Exp. & $\begin{array}{c}\text { \# Data } \\
\text { Streams }\end{array}$ & \# CPUs & $\begin{array}{c}\text { \# Output } \\
\text { Streams }\end{array}$ & \# Events & $\begin{array}{c}\text { Data Set } \\
\text { Size }\end{array}$ & $\begin{array}{c}\text { \# Reconstruct } \\
\text { Charm }\end{array}$ \\
\hline $1980-2$ & E-516 & 1 & 1 & 1 & $20 \mathrm{M}$ & 70 GBytes & 100 \\
$1984-5$ & E-691 & 2 & 1 & 1 & $100 \mathrm{M}$ & 400 GBytes & 10,000 \\
$1987-8$ & E-769 & 7 & 17 & 3 & $400 \mathrm{M}$ & 1,500 GBytes & 4,000 \\
$1991-2$ & E-791 & 8 & 54 & 42 & $20,000 \mathrm{M}$ & 50,000 GBytes & 200,000 \\
& & & & & & & \\
\hline
\end{tabular}

\subsection{Off-Line Computing Issues}

The trend toward more cost effective computing seems to be continuing at the same phenomenal rate as over the last decade. This implies that continuation of the general slope seen in Fig. 1 is possible from this point of view. However, even with these expected gains, additional improvements will be required to reach $10^{8}$ reconstructed charm decays. Again, the trigger is usually hoped to provide the answer. 


\section{Summary and Conclusions}

It is necessary to put all the above considerations together in proposing an experiment to achieve the goal of $10^{8}$ reconstructed charm decays. Using the example of a hadron beam experiment proves instructive. What happens if one tries to reach the goal? The flow is shown in Table III. A first pass through the conceptual design steps requires a return to the first step in an iterative procedure. It is not clear that one can reach the goal by simple extension of current concepts.

Table III.

\section{REACHING FOR $10^{8}$ RECONSTRUCTED CHARM DECAYS - SIMPLE HADRONIC EXTENSION?}

1. Reconstructed charm $=0.005 \times$ number of charm events

2. Charm events $=0.002$ of hadron induced interactions

3. $2-10 \%$ interaction length target hadrons

4. $3 \times 10^{6}$ seconds of beam spill

5. Suppose $100 \mathrm{MByte} / \mathrm{second}$ into DA pipeline

6. and 3 KBytes/event

7. Given the above lines 2 and 4

8. Given above lines 6 and 7

9. But, as trigger rejection must go from today's factor of 5

$$
\begin{aligned}
& \rightarrow \quad 2 \times 10^{10} \text { charm events } \\
& \rightarrow \quad 10^{13} \text { interactions } \\
& \rightarrow \quad 1-5 \times 10^{14} \text { inc. } \\
& \rightarrow \quad 30-160 \text { Mhz beam } \\
& \rightarrow \quad 30 \mathrm{~K} \text { events/second } \\
& \rightarrow \quad 3 \text { Mhz interaction rate } \\
& \rightarrow \quad \text { Trigger } R=100 \\
& \rightarrow \quad \text { Return to line } 1
\end{aligned}
$$

(Remember to reduce efficiency in line 1 when $R$ increases)

The basic question in my mind is whether a $10^{8}$ reconstructed charm decay experiment can be achieved by this simple extension of current techniques. A new technological breakthrough (akin to that provided by SMDs) is required. Furthermore, even with $10^{8}$ reconstructed decays, do we know how to extrapolate current precision and upper limits into the future? What would be the equivalent number of decays needed in terms of physics reach? Narrowing the physics goals to the most important ones will allow more incisive choices and better matched compromises.

For these reasons, I prefer to think about $10^{8}$ equivalent charm decays. That is, without a new breakthrough, I don't expect a generic, open geometry experiment whose physics reach is represented by extrapolation to $10^{8}$. We will need to narrow our focus, our experiment design, to 
particular physics goals. Then, it may be possible to achieve the desired physics sensitivity for those particular goals. Otherwise, we need something that breaks with recent tradition. Hopefully, the CHARM2000 workshop will point the way to the best choices of physics and experiment setup.

\section{Acknowledgements}

I am specially indebted to my colleagues on the series of charm experiments performed over the last 15 plus years at the Tagged Photon Laboratory. I want to mention specifically Gilvan Alves, Steve Bracker, Tom Carter, Catherine James, Paul Karchin, Simon Kwan, Lalith Perera, Milind Purohit, Marleigh Sheaff, Don Summers, Keith Thorne, Andrew Wallace, and Zhongxin Wu whose works I have specifically consulted in preparing this review. I also thank David Christian for use of his plot in Fig. 1.

\section{References}

1 D.C. Christian, private communication.

2 (E691 Collaboration) J. R. Raab et al.; Phys. Rev. D37, 2391 (1988).

3 E687, NA14 and E691 data summarized by A. Ali, DESY 93-105, Lectures given at the XXI International Meeting on Fundamental Physics, Miraflores de la Sierra, Madrid, 9-15 May, 1993; to appear in the Proceedings (Eds. F. Barreiro et al.).

4 NA27, NA32, E653 and E769 data on pions and NA27, E653, E743 and E769 data on protons summarized by A. Wallace et al., FERMILAB-Conf-94//84.

5 (E687 Collaboration), J. Cumalat and L. Moroni, in Proceedings of the CHARM2000 Workshop.

6 J. Martin et al, FERMILAB-CONF-81/40-EXP and CERN Microproc. 1981:164 (QCD201:T6:1981).

7 J. A. Appel, Europhysics Study Conf. on Search for Charm, Beauty and Truth, Erice, Sicily, Nov 15-22, 1981. Published in Erice EPS: Charm 1981:555 (QCD161:E86:1981). 